\title{
A guidance for assessing and communicating uncertainties
}

\author{
P.H.M. Janssen*, A.C. Petersen*, J.P. van der Sluijs ${ }^{\star *}$, J.S. Risbey ${ }^{\star * *}$ \\ and J.R. Ravetz ${ }^{* \star * *}$ \\ *Netherlands Environmental Assessment Agency (RIVM/MNP), P.O. Box 1, 3720 BA, Bilthoven, \\ The Netherlands (E-mail: Peter.Janssen@rivm.nl; Arthur.Petersen@rivm.nl) \\ ${ }^{\star *}$ Copernicus Institute for Sustainable Development and Innovation, Dept. of Science Technology and \\ Society, Utrecht University, The Netherlands (E-mail: j.p.vanders/uijs@chem.uu.nl) \\ ***School of Mathematical Sciences, Monash University, Clayton, Australia \\ (E-mail: james.risbey@sci.monash.edu.au) \\ ${ }^{\star \star \star \star}$ Research Methods Consultancy (RMC), London, UK (E-mail: jerry_ravetz@lineone.net)
}

\begin{abstract}
In the daily practice of science for policy, as experienced by governmental agencies which inform the policy and the public on the state and outlook of the environment, there is a pressing need for guidance in assessing and communicating uncertainties. This need extends beyond the quantitative assessment of uncertainties in model results, and focuses on the entire process of environmental assessment, running from problem framing towards reporting the results of the study. Using the Netherlands Environmental Assessment Agency (RIVM/MNP) as a case, the development, structure and content of such a guidance system is highlighted. Conditions for a successful implementation of the guidance system are discussed, and some prospects for future work are outlined.
\end{abstract}

Keywords Communication; guidance; interaction; science-policy; tool catalogue; uncertainty assessment

\section{Introduction}

At the onset of 1999, the Netherlands National Institute for Public Health and the Environment (RIVM) was faced with a credibility crisis due to public criticism in a Dutch quality newspaper by an employee of the RIVM. He criticized the institute for suggesting an unjustified level of certainty in reporting environmental studies, by not duly accounting for uncertainty and relying too much on the virtual reality of poorly validated models. His criticism attracted much media attention in the Netherlands and triggered extensive public and political debate on the credibility, reliability and quality of environmental statistics and model-based environmental foresight, as well as on the role and position of science in policy-making (van der Sluijs, 2002).

This event can be seen as typical for the role and position of science for policy and society in a world which is becoming increasingly interlinked and complex. Now decisions are urgent, stakes are high and diverse, values are in dispute, uncertainty and ignorance involved are high, and trust is fragile (Funtowicz and Ravetz, 1999). All these problems are common for sustainability, risk and safety issues. The changing relationship between science, policy and society calls for processes and arrangements where issues such as transparency and novel forms of quality control (e.g., extended peer review), public participation, multiple perspectives, reflexivity, transdisciplinarity and accountability are at the forefront in establishing knowledge that is more socially robust (Nowotny et al., 2001).

At RIVM, the above-mentioned credibility crisis was the impetus for developing a system of guidance for assisting its employees in their daily practice of performing research to advise policy-makers and the public on the state and outlook of the environment, placing special focus on the assessment and communication of uncertainties. In this 
paper the development of this guidance system is described, highlighting its major parts. We will end with discussing conditions for its successful implementation and outlining prospects of future work.

\section{On the development of the guidance system}

After the media affair in 1999 a national and international review of the RIVM's environmental assessment activities was undertaken, leading to the development of a guidance system for uncertainty assessment and communication in environmental assessment studies. It was judged that the scope of the guidance system should extend beyond the mere quantitative assessment of uncertainties in model results per se, and should focus instead on the entire process of environmental assessment. It should explicitly address issues such as problem framing, stakeholder participation, indicator selection, appraisal of the knowledge base, mapping and assessment of relevant uncertainties, and reporting of the uncertainty information, since these are potential key aspects in identifying and communicating uncertainties. Moreover the guidance system should provide a prioritized list of uncertainty types and sources that need particular attention for the case at hand, in view of its societal context and the function of the assessment. The system is intended to give advance warnings of which bottlenecks can occur with respect to dealing with these uncertainties and what additional effort should then be made in the field of uncertainty assessment. Finally, it should offer advice on the selection of quantitative and qualitative methods and tools to adequately estimate uncertainties in the given context and to communicate them to scientific researchers, the clients (usually ministries), other actors in the policy process, and the broader public.

Commissioned by RIVM, the development of the guidance system started in September 2001, under the leadership of Dr. van der Sluijs in close cooperation with RIVM and with a number of international uncertainty management specialists. In October 2001, an expert workshop was held to obtain input and feedback from the uncertainty management experts on a first sketch of the guidance system. This led to a draft version, in the form of a detailed questionnaire, which was subsequently presented to employees of the RIVM in a user workshop in November 2001. Though considered generally as a very thorough basis for uncertainty assessment, the detailed guidance document was judged by many of the users as being too comprehensive to be easily applicable in all cases. They preferred a shorter, pragmatic, easy-to-use version which could be applied at varying depths/levels, and which would offer specific hints and suggested actions on dealing with uncertainty. Therefore it was decided in 2002 to develop a concise mini-checklist covering the major points in mapping and communicating uncertainties, as well as an associated quickscan version, which includes hints and preferred actions.

All this resulted in a suite of components (Figure 1), called the RIVM/MNP Guidance for Uncertainty Assessment and Communication, denoted by Guidance for short in the following. The Guidance can be consulted in various stages of the environmental assessment process by various users at a frequency and level which suits their individual needs best. For instance, at the beginning of a project, the guidance can play an important role in designing and elaborating the way uncertainty will be dealt with during the project. During a project, it can be of assistance in performing the uncertainty assessment and communicating the results. After a project, it can be of use in reviewing and evaluating the project. The group of intended users of the Guidance covers a large fraction of the employees of the Netherlands Environmental Assessment Agency ${ }^{1}$ (RIVM/MNP) 


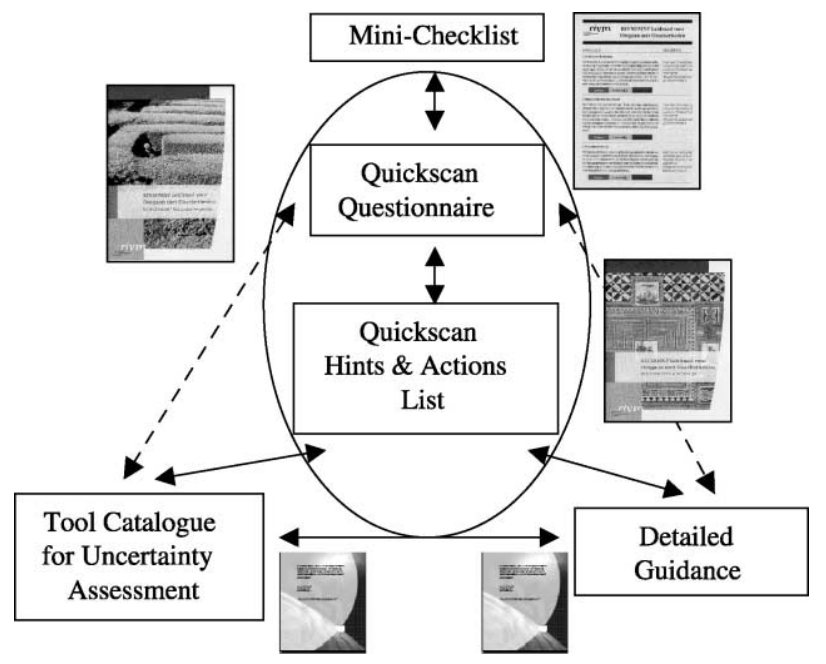

Figure 1 Structure of the RIVM/MNP guidance for uncertainty assessment and communication

(e.g. project leaders, project-team members, researchers or policy advisers), as well as others (e.g. stakeholders involved in an extended peer-review of the project). Project leaders will typically use those components of the Guidance which are at a high level of aggregation (the Mini-Checklist and the Quickscan), while project-team members, researchers and policy advisers will more often also take up parts of the more detailed Guidance.

The interrelationships between the components that constitute the Guidance is as follows (Figure 1): The 'Mini-Checklist' (Petersen et al., 2003) is a back-of-the-envelope kind of tool which can also serve as a portal to the other components of the Guidance. By concisely presenting the potentially important issues in the various stages of the environmental assessment process - running from problem framing to reporting the results - it functions as a reminder list and instrument for reflection on the (desired) way of dealing with uncertainties and value-loadings in providing policy advice. It renders a brief account of the way uncertainty has been dealt with, and points to the 'Quickscan Questionnaire' (Petersen et al., 2003) if further elaboration is desired. This latter document (optionally) refers the user to the 'Quickscan Hints \& Actions List' (Janssen et al., 2003) which has an advisory function and describes possible implications of the answers given to the questions in the Quickscan Questionnaire.

Some of the hints and actions point directly to parts of the 'Detailed Guidance' (van der Sluijs et al., 2003) and the 'Tool Catalogue for Uncertainty Assessment' (van der Sluijs et al., 2004). These documents can be considered as basic components underlying the complete Guidance System. Although the Quickscan documents are presented as autonomous components which can be used in a separate mode, they are intrinsically related to these basic components. The 'Detailed Guidance' has been set up as an elaborate questionnaire for a deeper analysis of various aspects of dealing with uncertainty. It also contains a glossary of terms related to uncertainty assessment and communication. The associated 'Tool Catalogue for Uncertainty Assessment' offers information on different quantitative and qualitative methods and tools that can be utilized to assess uncertainties. The above documents are freely available on the Internet (www.nusap.net).

The ultimate decision which components of the Guidance to use largely depends on the importance, the nature and the level of the uncertainties in the assessment concerned 
and on the resources available. Inevitably this decision is subjective to a certain extent and is influenced by the intentions and interests of the specific user(s): e.g. stakeholders will focus on other issues than project-team members or project leaders. In fact these different foci are brought into the open by the use of the Guidance. The mini-checklist and the quickscan components are the parts of the Guidance that will be used most frequently. If a user has the time and the mandate, then the detailed Guidance will be used to supplement and deepen the analysis. The mini-checklist and quickscan are discussed in below.

\section{Structure of mini-checklist and quickscan documents}

The mini-checklist concisely covers six central uncertainty-related themes in the environmental assessment process, including problem framing, stakeholder participation, selection of indicators, appraisal of the knowledge base, mapping and assessment of relevant uncertainties, reporting of the uncertainty information. It asks the user to reflect explicitly on how these issues are dealt with in the study at hand. The quickscan documents, consisting of a questionnaire and an associated hints and action list, elaborate this in more detail. In the following we will highlight point by point the six central themes addressed in these documents from the perspective of a user who is performing the environmental assessment.

\section{Problem framing}

In this stage, the problem and its context and history are outlined, by identifying major issues, past work, the level of contention and the (expected) role of the assessment in the policy or decision making process. The user is explicitly asked to consider various views/perspectives on the problem, and to pay attention to the problem's interconnectedness with other problems. He/she is asked to be specific on what knowledge is needed with regard to the problem, and into which research questions this is translated. Possibly relevant aspects which are not dealt with in these research questions have to be indicated. Moreover, it should be outlined what role the study is expected to play in the policy process, and what the relation is with previous studies on the subject (policy context and problem history).

\section{Involvement of stakeholders}

This step concerns the identification of the main parties (stakeholders/actors) and their views and roles with respect to the problem, as well as the aspects of the problem about which they disagree. The problem at hand is characterised in terms of a number of features: level of dissensus on policy goals regarding the problem, type of knowledge needed, and the decision stakes and uncertainties involved. All this information helps in deciding on an appropriate level and form of stakeholder participation for the current study.

\section{Selection of indicators}

In environmental assessments, the relevant features of the problem under study are typically expressed in terms of indicators or target variables. Selection of indicators is therefore an important step in shaping a study, and it is important to substantiate the final choices, discussing their shortcomings and associated controversies as well. This involves judging how well the selected indicators address key aspects of the problem as it has been framed, and how much support there is among scientists and within society (including decision-makers/politicians) for the use of these indicators for the problem at hand. Moreover, there should be an examination of how to deal with a potential lack of support, giving attention to differences in views and interest, and specifying what the 
consequences of these differences will be for the meaning and value of the study. Consider giving the stakeholders a role in defining or revising indicators.

\section{Appraisal of knowledge base}

This stage is concerned with answering the question of the adequacy of the available knowledge base for the assessment. It involves questions like: What quality criteria are relevant for answering the research questions? What knowledge and methods are needed to obtain answers of the required quality? What are the most important bottlenecks in the way of achieving this, in the light of existing controversies and weaknesses in the knowledge base? What will be the effect on the quality of the results, and which actions should be taken to clear these bottlenecks? In this way the user gets useful information for (re)shaping the study, in consultation with the client, and for adequately focusing the assessment and its reporting.

\section{Mapping and assessment of the relevant uncertainties}

In this step, the user is asked to identify the uncertainties most relevant to the problem, and to estimate what effort will be required to map these uncertainties adequately, providing information on their extent, nature (being epistemic or stochastic) and location. Moreover the possible consequences of the uncertainties for the conclusions of the study have to be indicated, and an indication should be given on how to assess the most important uncertainties and their consequences, within the limitations of the available resources (time, money, people, expertise, etc.). The actual planning and performing of the uncertainty assessment completes this step.

To support the user in these tasks various hints and tools are provided (c.f. Janssen et al. 2003, van der Sluijs et al. 2003, 2004). For identifying the most relevant uncertainties, the uncertainty matrix presented in Table 1 can be used. This heuristic device is an adapted and extended version of the matrix proposed in Walker et al. (2003) to classify and report important dimensions of uncertainty. A tool catalogue is provided (van der Sluijs et al. 2004) to assist the user in choosing appropriate methods for dealing with the identified uncertainties. In this document comprehensive information is given on various quantitative and qualitative uncertainty assessment techniques (global sensitivity analysis, NUSAP, expert elicitation, scenario analysis, model quality assessment, etc.). The presented information concerns a brief description of the specific technique and its goals, strengths and limitations, required resources, as well as guidelines for its use and warnings for typical pitfalls. It is supplemented by references to handbooks, software, example case studies, websites, experts, etc. The tool catalogue is a 'living document,' which will be made available on the web in the future, and to which descriptions of additional tools can be added.

Table 1 Uncertainty Matrix (cf. Janssen et al., 2003; Walker et al., 2003)

\begin{tabular}{|c|c|c|c|c|}
\hline \multirow{2}{*}{$\begin{array}{l}\text { Location of } \\
\text { uncertainty } \\
\qquad \downarrow\end{array}$} & $\begin{array}{l}\text { Level of uncertainty (from } \\
\text { determinism, through probability and } \\
\text { possibility, to ignorance) }\end{array}$ & \multirow{2}{*}{\begin{tabular}{|l|}
$\begin{array}{l}\text { Nature of } \\
\text { uncertainty }\end{array}$ \\
$\begin{array}{l}\text { Epis- I Varia- } \\
\text { temic }\end{array}$ bility \\
\end{tabular}} & $\begin{array}{l}\text { Qualification } \\
\text { of knowledge } \\
\text { base }\end{array}$ & $\begin{array}{l}\text { Value- } \\
\text { ladenness } \\
\text { of choice }\end{array}$ \\
\hline & $\begin{array}{l}\text { Statistical I Scenario I Recognication } \\
\text { Uncertainty } \\
\text { Uncertainty }\end{array}$ & & $-\begin{array}{lll} & 0 & \text { I } \\
& \mathbf{1} & \mathbf{1} \\
\end{array}$ & $-\quad \mathbf{I O}^{0} \mathbf{I}+$ \\
\hline Context & & & & \\
\hline Expert judgment & 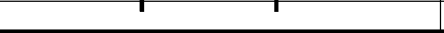 & † & 1 & \\
\hline Structure & $\mathbf{T}$ & $\mathbf{T}$ & $\mathbf{T}$ & $\mathbf{T}$ \\
\hline Implementation & I & I & $\mathbf{I}$ & I \\
\hline Parameters & 1 & I & I & \\
\hline Inputs & & & & \\
\hline Data & & & & \\
\hline Outputs & & & & \\
\hline
\end{tabular}




\section{Reporting of uncertainty information}

Reporting of uncertainty information preferably takes place during the whole environmental assessment process, not only at the final delivery of results. In this communication it is necessary to be aware of (i) the context of the reporting (why, to whom, on behalf of whom, when, where) and (ii) the robustness of the main messages for uncertainties in the knowledge base and for any deviations from the employed assumptions and choices. Reporting on the policy-relevant uncertainties and their possible consequences for policy making, politics and society should take place in a clear manner, tailored to the intended audience(s). In written reporting, the results should be presented in a balanced and consistent way, providing a traceable account and adequate backing of the presented material. For example, this can be achieved by offering the information in a step-wise fashion. Taking account of the fact that readers often scan a text selectively it is recommended to present important uncertainty information explicitly at strategic points, e.g., in the introduction, conclusions, summary, and text-boxes.

\section{Conclusions}

The foregoing illustrates that the guidance system provides structure to the task of uncertainty management and can be employed in a flexible way. The Guidance stimulates reflection and deliberation on how uncertainties are (to be) handled and communicated effectively and helps to avoid pitfalls. Tools for uncertainty assessment are made more easily available, and can be selected in a more tailored manner, on basis of problem characteristics. Although the guidance system was initially developed in the context of environmental assessments, it can be applied in other application areas as well, with some minor adaptations as appropriate.

For its success, some conditions are essential. Firstly, the commitment of higher management is crucial, since time and effort spent on dealing with uncertainties must be considered as relevant. By its primary focus on the policy-relevance of uncertainties, the Guidance puts this issue explicitly to the fore. Secondly, it would certainly help if the use of the Guidance was included as standard activity in the prevailing project management. At RIVM/MNP this has been established by including the Guidance formally in the quality assurance system. Thirdly, its further application and institutionalization will require motivating and training the potential users of the Guidance, showing them the benefits of its use. Currently training sessions in the use of the Guidance are organized for employees of the RIVM/MNP. To support the introduction in daily practice, a web-based version of the Guidance is under construction, providing quick and easy access to its various parts. We hope to stimulate this introduction process further by providing appropriate uncertainty assessment tools, and by gradually building an expertise network and a 'good-practice examples' data-base in using the Guidance. Moreover a styleguide for uncertainty communication is in preparation.

In fact, we currently are only at the start of implementing the presented systematic ideas of uncertainty management in an institutional setting. The above-mentioned activities must therefore be considered as first steps. In due time - after its fuller implementation - the use of the Guidance will be evaluated, leading possibly to further adaptations. For the time being we can already identify two major issues which will deserve future attention. The first one refers to the further deployment and development of the tool catalogue with methods for: (i) propagating and analyzing qualitative and semi-quantitative uncertainty information (e.g., concerning value-loadings, assumptions, pedigree scores), especially in expert-reasoning and model-based calculation 'chains'; and for (ii) synthesizing qualitative and (semi)-quantitative uncertainty information. The 
interactions in order to find suitable arrangements and forms for knowledge production and uncertainty communication, thus enhancing the effective use of science for policy or society.

Notwithstanding that there is still a long way to go, one can consider the Guidance with its specific focus on problem context and socio-political embedding, accountability, transparency and reflexivity, participation and extended peer review - as a useful contribution towards new social practice of science in a postmodern era, as exemplified by e.g. the post-normal science and mode 2 science paradigms (Funtowicz and Ravetz, 1999; Nowotny et al., 2001).

\section{Acknowledgements}

We acknowledge the help of many colleagues from RIVM/MNP and various uncertainty experts who have aided in the development of the Guidance by written contributions or by discussions, comments and reviews.

\section{References}

Funtowicz, S. and Ravetz, J. (1999). Post-Normal Science - an insight now maturing. Futures, 31, 641-646.

Janssen, P., Petersen, A., van der Sluijs, J., Risbey, J. and Ravetz, J. (2003). RIVM/MNP Guidance for Uncertainty Assessment and Communication: Quickscan Hints \& Actions List, RIVM/MNP, Bilthoven, The Netherlands, ISBN 90-6960-105-2.

Nowotny, H., Scott, P. and Gibbons, M. (2001). Re-Thinking Science. Knowledge and the Public in an Age of Uncertainty, Polity Press, Cambridge.

Petersen, A., Janssen, P., van der Sluijs, J., Risbey, J. and Ravetz, J. (2003). RIVM/MNP Guidance for Uncertainty Assessment and Communication: Mini-Checklist \& Quickscan Questionairre, RIVM/MNP, Bilthoven, The Netherlands, ISBN 90-6960-105-1.

van der Sluijs, J. (2002). A way out of the credibility crisis of models used in integrated environmental assessment. Futures, 34, 133-146.

van der Sluijs, J., Risbey, J., Kloprogge, P., Ravetz, J., Funtowicz, S., Corral Quintana, S., Guimarães Pereira, Â, De Marchi, B., Petersen, A., Janssen, P., Hoppe, R. and Huijs, S.W.F. (2003). RIVM/MNP Guidance for Uncertainty Assessment and Communication: Detailed Guidance. Report nr: NWS-E-2003-163. Utrecht University, Utrecht, The Netherlands.

van der Sluijs, J., Janssen, P., Petersen, A., Kloprogge, P., Risbey, J., Tuinstra, W. and Ravetz, J. (2004). RIVM/MNP Guidance for Uncertainty Assessment and Communication: Tool Catalogue for Uncertainty Assessment. Report nr: NWS-E-2004-37. Utrecht University, Utrecht, The Netherlands.

Walker, W., Harremoës, P., Rotmans, J., van der Sluijs, J., van Asselt, M. Janssen, P. and Krayer von Krauss M. (2003). Defining uncertainty: a conceptual basis for uncertainty management in model-based decision support. Integrated Assessment, 4(1), 5-17. 